\title{
Neuronally-Enriched Exosomal miR-27b Reveals Mechanism for Ibuprofen-Induced Acute Effects on Reward-Related Brain Processing in a Randomized, Placebo-Controlled, Double-Blind Trial
}

Kaiping Burrows ( $\boldsymbol{\nabla}$ kburrows@laureateinstitute.org )

Laureate Institute for Brain Research

\section{Leandra K. Figueroa-Hall}

Laureate Institute for Brain Research

Rayus Kuplicki

Laureate Institute for Brain Research

Jennifer L. Stewart

Laureate Institute for Brain Research

Ahlam M. Alarbi

University of Oklahoma Health Sciences Center

Rajagopal Ramesh

University of Oklahoma Health Sciences Center

Jonathan B. Savitz

Laureate Institute for Brain Research

T. Kent Teague

University of Oklahoma Health Sciences Center

Victoria B. Risbrough

University of California, San Diego

Martin P. Paulus

Laureate Institute for Brain Research

\section{Research Article}

Keywords: extracellular vesicles, neuronally-enriched exosomes, MiR, miR-27b, miR-320b, ibuprofen, reward-related brain processing, monetary incentive delay task, fMRI

Posted Date: August 17th, 2021

DOI: https://doi.org/10.21203/rs.3.rs-803515/v1 
License: (c) (i) This work is licensed under a Creative Commons Attribution 4.0 International License. Read Full License 


\section{Abstract}

This double-blind, randomized, repeated measures study evaluated whether acute administration of an anti-inflammatory drug modulates neuron-specific, inflammation-modulating microRNAs linked to macroscopic changes in reward processing. Twenty healthy subjects ( 10 females, 10 males) completed a monetary incentive delay (MID) task and provided blood samples after administration of placebo, 200 $\mathrm{mg}$, or $600 \mathrm{mg}$ of ibuprofen. Neuronally-enriched exosomal microRNAs were extracted from plasma and sequenced. Results showed that: (1) $600 \mathrm{mg}$ of ibuprofen exhibited higher miR-27b-3p, miR-320b, miR23b and miR-203a-3p expression than placebo; (2) higher mir-27b-3p was associated with lower insula activation during MID loss anticipation; and (3) there was an inverse relationship between miR-27b-3p and MID gain anticipation in bilateral putamen during placebo, a pattern attenuated by both $200 \mathrm{mg}$ and $600 \mathrm{mg}$ of ibuprofen. Our findings indicate that miR-27b could be an important messaging molecule altered by anti-inflammatory drugs that relates to the processing of positive or negative valenced information.

\section{Introduction}

Inflammatory processes may play a significant role in psychiatric disorders in general ${ }^{1}$, and in mood disorders in particular ${ }^{2}$. Epidemiological data suggest an overlap between depression and inflammatory illnesses ${ }^{3,4}$, and prospective studies show that chronic inflammation increases depression risk ${ }^{5}$. There are cross-sectional associations with inflammatory mediators in major depressive disorders (MDD) such that depressed individuals exhibit higher levels of circulating proinflammatory cytokines, such as interleukin-6 (IL-6) and tumor necrosis factor (TNF ${ }^{6,7}$. One core feature of a major depressive episode is anhedonia loss of interest or pleasure in nearly all rewarding activities. Previous research suggests that elevated peripheral inflammation is associated with decreased brain striatal activity during reward prediction and anticipation in $\mathrm{MDD}^{8,9}$. Here, we investigate whether ibuprofen, a non-steroidal, anti-inflammatory drug (NSAID), will alter neuronally-enriched exosomes (NEE), inflammatory-related microribonucleic acids (miRs), and modulate reward-related brain processing in healthy participants. Understanding ibuprofen's role in its ability to modulate inflammatory-related regulatory miRs and reward processing may elucidate potential treatment targets for depressed subjects with high inflammation to improve their anhedonic symptoms.

Clinical trials have investigated the antidepressant effects of NSAIDs, including selective cyclooxygenase (COX) inhibitors such as ibuprofen, given their strong anti-inflammatory properties ${ }^{10}$. Ibuprofen drives non-selective, reversible inhibition of COX enzymes, COX-1 and COX-2 ${ }^{11}$. However, ibuprofen's exact mechanism of action is still under investigation with respect to the COX-independent effects on $\beta$-catenin, nuclear factor kappa-light-chain-enhancer of activated B cells (NF-kB), peroxisome proliferator-activated receptor gamma (PPARY), and p53-dependent molecular pathways ${ }^{12}$. Ibuprofen's effect on the central nervous system (CNS) may not be limited to its ability to inhibit COX-1 and prostaglandin (PG) 
synthesis ${ }^{11}$. In particular, Ibuprofen activates the transcription factor PPARy in neuronal-like cells ${ }^{13}$, which is proposed as a potential mechanism for neural tissue maintenance ${ }^{14}$.

While ibuprofen is one of the most commonly used and prescribed NSAIDs, few research studies have examined ibuprofen's effect on the brain in human participants using neuroimaging and those that have exclusively focused on pain-related processing. In one study by Pizzi et al., ten healthy subjects underwent a double-blind, placebo-controlled, randomized, cross-over pharmacological functional magnetic resonance imaging (phFMRI) study with painful somatosensory stimulation of the right median nerve. Authors reported a task-related increase of blood oxygen level dependent (BOLD) signal between drug and placebo in the primary somatosensory area that was not related to changes in subjective pain scores $^{15}$. Another study using ibuprofen ( $600 \mathrm{mg}$ single dose) failed to suppress the secondary mechanical hyperalgesia-evoked neural response in a region of the brainstem's descending pain modulatory system (right nucleus cuneiformis) and left (contralateral) posterior insular cortex and secondary somatosensory cortex ${ }^{16}$. Thus, while there is some evidence of pain-related processing changes in the brain, there is no study examining the effects of ibuprofen on reward-related processing.

MiRs comprise a large family of small, non-coding RNAs consisting of 18-25 nucleotides in length that act as key posttranscriptional regulators of gene expression. MiRs are predicted to control the transcription of approximately $50 \%$ of all protein-coding genes and are, therefore, an important class of epigenetic regulators ${ }^{17}$. miR-27b, an intragenic miR on chromosome 9 clustering with miR-23b and miR24-1 in humans ${ }^{18}$, is among the strongest regulators of lipid metabolism ${ }^{19,20}$ and adipogenesis ${ }^{21}$. Moreover, miR-27b is robustly increased after a cytomegalovirus infection ${ }^{22}$, attenuates inflammatory mediators, such as NF-KB activity, IL- $6^{23}$ and transcription of inflammatory target genes ${ }^{24}$, shifts toward the anti-inflammatory state in endothelial cells via cell cycle inhibitory and vasculoprotective effects ${ }^{25}$, and is involved in glucose transport and insulin regulation ${ }^{26}$. miR-27b also has many regulatory functions with targets including PPARy and PPARa ${ }^{27}$, a subfamily of nuclear receptor proteins and key regulators of inflammation, which may also be potential targets for ibuprofen. As such, Fig. 1A depicts mir-27b's role in PPARy regulation. mir-27b increases PPARy expression, resulting in: (1) inhibition of several transcription factors, such as NF-kB and AP-1, and (2) attenuation of proinflammatory mediators including IL-6 and TNF. miR-27b also regulates other gene targets, such as TGFB1, MMP13, ST14, and SEMA6A, which have roles in inflammatory processes, extracellular matrix degradation, and cell-cell signaling as shown in Fig. $\mathbf{1 B}^{28}$. miR-27b is significantly expressed in the brain, upregulates neuronally expressed targets and plays a role in both excitatory and inhibitory neurotransmission through the modulation of genes involved in regulation of glutamate and gamma aminobutyric acid (GABA) metabolism and transport ${ }^{29}$.

In comparison, miR-320b is an intragenic miR on chromosome 1 that has been implicated in neuronal differentiation ${ }^{30,31}$, regulation of inflammatory processes via the nucleotide-binding oligomerization domain $^{32}$, and regulation of endoplasmic reticulum stress ${ }^{33}$. miR-320b is robustly increased in anterior cingulate cortex and habenula of individuals with $\mathrm{MDD}^{34}$, regions of the brain thought to be important for 
pain affect and processing ${ }^{35,36}$. Figure 2 shows additional gene targets for miR-320b including DTNA, NUMB, MDFIC, TVP23A, and SEC14L1, which are responsible for functions such as formation and stability of synapses, local repair of brain ventricular wall damage, WNT and JNK signaling, vesiclemediated transport, and innate immune-mediated antiviral inhibition ${ }^{37}$. Both miR-27b and miR-320 b are expressed in the brain, affect a range of metabolic, inflammatory, and pain processes and are, in turn, modulated by anti-inflammatory drugs such as ibuprofen. These miR molecular signatures and miRmediated functions can be experimentally investigated through the enrichment of extracellular vesicles (EVs), with a focus on brain-specific EVs.

EVs are comprised of a diverse set of particles that include exosomes, microvesicles, and apoptotic bodies that participate in intercellular communication and in paracrine and endocrine signaling ${ }^{38}$. In particular, EVs mediate complex and coordinated communication among neurons, astrocytes, and microglia, both in the healthy and diseased brain ${ }^{39}$. NEEs in the CNS cross the blood brain barrier into the blood (Fig. 3) and peripheral exosomes from the blood and organ systems (heart, liver, and thymus, among others) can also cross into the CNS. MiRs make up a high percentage of the specialized cargo contained in exosomes and mediate their biology, function, and therapeutic potential ${ }^{40,41}$, supporting the remarkable ability of MiRs to communicate between cells and regulate protein expression across species ${ }^{42}$. The immune response mediated by microglial-derived exosomes is most prominently involved in the spread of neuroinflammation, neurodegenerative disorders, and brain cancer as shown with the use of conditioned media from primary and immortalized rodent brain microglial cells $s^{43-45}$. Emerging evidence suggests miRs contained in EVs are an important component in regulating critical pathways in the brain associated with MDD, amyotrophic lateral sclerosis, Alzheimer's disease, and Parkinson's disease, which share several neuroinflammatory-associated processes ${ }^{46}$. While brain-specific EVs from psychiatric and neuroinflammatory conditions can point to dysregulated molecular signatures and pathways, the experimental investigation of brain-specific EVs in healthy controls can also give insight into homeostatic processes and changes mediated by administration of anti-inflammatory pharmacologics.

This investigation aimed to integrate several experimental approaches to provide evidence that an acute administration of an anti-inflammatory drug modulates EV cargo, and more specifically, NEE-specific, inflammatory-modulating miRs that are linked to macroscopic changes in brain function - reward processing, in particular. Specifically, we conducted a pharmaco-phfMRI study of acute administration of placebo, 200 milligrams (mg), or $600 \mathrm{mg}$ ibuprofen in healthy volunteers during the MID task ${ }^{47}$. Subsequently, we isolated exosomes from blood samples, enriched them for NEEs, and performed an RNAseq analysis focusing on miRs implicated in ibuprofen-regulated pathways. Lastly, we conducted a moderator analysis to determine whether the brain activation changes induced by ibuprofen were modulated by the level of miRs obtained from NEEs. Given the link between inflammatory mediators and reward processing 9,48,49, we hypothesized that inflammatory pathway-related miRs should moderate the ibuprofen-related brain activation changes in striatal regions. 


\section{Methods}

Participants. This study was conducted at the Laureate Institute for Brain Research (LIBR) in Tulsa, Oklahoma (OK) between 6/30/2015 and 10/30/2015. The study was approved by the Western Institutional Review Board, and all experiments were performed in accordance with the Declaration of Helsinki; Informed consent was obtained from all participants. Participants were recruited from the general community through newspaper, flyer, radio and other media advertisements in Tulsa and the surrounding regions of OK. Subjects were screened by trained clinical interviewers to evaluate the following study exclusion criteria: (1) history of any mental health disorder such as dysthymia, simple phobia, MDD, obsessive compulsive disorder or panic disorder as a primary diagnosis currently or within 6 months prior to the screening visit; (2) history of schizophrenia, schizoaffective disorder, or a bipolar disorder; (3) current or past 6-month alcohol or drug abuse; (4) regular use (>15 days for past 30 days) of NSAIDS; (5) history of clinically significant hepatic cardiac, renal, neurologic, cerebrovascular, metabolic, gastric, or pulmonary disease; (6) past-year use of psychotropic drugs or antidepressants; (7) history of seizure disorders (except for childhood febrile seizures); (8) serious suicidal ideation or behavior; (9) women currently pregnant or planning to become pregnant within the next 18 weeks; (10) women currently menstruating; (11) claustrophobia, or phobia for injections or blood; and (12) fMRI-related exclusion criteria (e.g., medications treating cardiovascular, respiratory, endocrine and neurological diseases likely to influence cerebral blood flow). The trial was stopped after completing target recruitment. Twenty subjects ( 10 females, 10 males; mean age $=32$ years, $S D=7$, range $=27$ to 51 ; mean body mass index $\left[\mathrm{kg} / \mathrm{m}^{2}\right]=27, \mathrm{SD}=6$, range $=20.4$ to 44.7 ) completed this study (Table 1 ) (two additional subjects withdrew prior to study completion). See supplemental material for complete consort diagram. The sample size was determined based on prior ph-fMRI studies (e.g., Aupperle et al., 2012 (50)). 
Table 1

Sample Characteristics

\begin{tabular}{|c|c|c|c|c|}
\hline Variable & Training Dataset & Ibuprofen Dataset & Cohen's $d$ or $\chi^{2}$ & $p$ value \\
\hline$N$ & 480 & 20 & & \\
\hline Age (Years) & $34.5 \pm 10.5$ & $32.4 \pm 6.7$ & 0.274 & $<0.01$ \\
\hline Sex $=$ Male $(\%)$ & $217(45.2)$ & $10(50.0)$ & 0.04 & 0.85 \\
\hline Body Mass Index $\left(\mathrm{kg} / \mathrm{m}^{2}\right)$ & $26.5 \pm 5.5$ & $26.6 \pm 5.8$ & 0.12 & 0.37 \\
\hline Ethnicity & & & 0.08 & 0.77 \\
\hline Hispanic or Latino & 27 & 2 & & \\
\hline Not Hispanic or Latino & 437 & 18 & & \\
\hline Refused/do not know & $4 / 14$ & $0 / 0$ & & \\
\hline Educational Status & & & 0.19 & 0.56 \\
\hline Grandes 1-11 & 1 & 0 & & \\
\hline 12th grade (no diploma) & 6 & 0 & & \\
\hline Regular high school diploma & 40 & 0 & & \\
\hline GED or alternative credential & 229 & 0 & & \\
\hline Some college, no degree & 142 & 3 & & \\
\hline Associate degree & 51 & 11 & & \\
\hline Bachelor's degree & 1 & 2 & & \\
\hline Master's degree & 0 & 2 & & \\
\hline Missing data & 10 & 2 & & \\
\hline
\end{tabular}

Study Design. This double-blind, randomized, repeated-measures study was registered on clinicaltrials.gov (Identifier: NCT02507219, Study of Ibuprofen Effects on Brain Function, first posted date: $23 / 07 / 2015)$. After the screening visit (T0), eligible subjects were tested three times (T1, T2 and T3) and at each visit received placebo, $200 \mathrm{mg}$ or $600 \mathrm{mg}$ dose of oral ibuprofen (dose order was counterbalanced across subjects). Ibuprofen and visually identical placebo capsules were produced by a local compounding pharmacy in Tulsa, OK. The random allocation sequence was generated using a random number generator by a statistician not involved in data collection. Drugs were labelled $A / B / C$ and all study personnel were blinded until after data collection was complete. On the day of sessions T1, T2 and T3, subjects fasted overnight and arrived in the morning and received a snack along with either 
placebo, $200 \mathrm{mg}$ or $600 \mathrm{mg}$ of ibuprofen. Subjects underwent a fMRI scan approximately 1 hour after dosing and a blood draw approximately 5 hours after drug administration.

Blood samples. Venous blood was collected in BD Vacutainer Serum Blood Collection tubes with spraycoated silica as a clot activator and then transported to the University of Oklahoma Integrative Immunology Center (IIC) within two hours of collection. Blood tubes were centrifuged at $1300 \mathrm{xg}$ for 10 minutes at room temperature, serum was removed, aliquoted, and then stored at $-80^{\circ} \mathrm{C}$ until analysis.

Total exosomes (TEs) isolation. Total exosomes (TEs) were isolated from 250 microliters $(\mu \mathrm{L})$ serum samples using $63 \mu \mathrm{L}$ of ExoQuick exosome precipitation solution (System Biosciences, CA, United States; Catalog \#EXOQ5A-1). TE pellets were resuspended in $300 \mu \mathrm{L}$ of $1 \mathrm{X}$ phosphate buffered saline (PBS) (Thermo Fisher Scientific, United States; Catalog \#AM9625) with Halt protease and EDTA-free phosphatase inhibitor cocktail (Thermo Fisher Scientific, United States; Catalog \#78425). TEs were used immediately or stored at $-80^{\circ} \mathrm{C}$ until immunochemical enrichment of exosomes from neural sources could occur.

NEEs isolation. TEs were enriched by a magnetic streptavidin bead immunocapture kit against the neural adhesion marker, L1CAM (CD171) biotinylated antibody (Fig. 4A). This technology to enrich NEEs in blood samples has been previously validated ${ }^{61-63}$. The CD171 (L1CAM, neural adhesion protein) marker was used for NEE enrichment due to its high and relatively specific expression in neurons and low levels of expression in many other cell types (neuronal marker assessments show that majority of the exosomes in NEEs have a neuronal origin ${ }^{61}$, and the level of neuronal markers neurofilament-light (NF-L) and synaptophysin (SYP) are significantly enriched in NEE by 86 -fold and 951 -fold compared to TEs ${ }^{64}$ ). Briefly, $80 \mu \mathrm{L}$ of $9.1 \mu \mathrm{m}$ diameter covalently cross-linked streptavidin magnetic beads (System Biosciences, CA, United States; Catalog \#CSFLOWBASICA-1) and $80 \mu \mathrm{L}$ of 100 nanograms $/ \mu \mathrm{L}$ of mouse anti-human CD171 biotinylated antibody (clone 5G3, eBioscience, United States; Catalog \#13-1719-82) were incubated on ice for 2 hours with gentle flicking every 30 minutes. After washing three times in $1 \mathrm{X}$ Bead Wash Buffer (BWB) (Systems Biosciences, CA, United States; Catalog \#CSFLOWBASICA-1) using a magnetic stand, the bead/antibody complex was suspended in $400 \mu \mathrm{L}$ of BWB. $200 \mu \mathrm{L}$ of TE suspensions were added to the bead/antibody complex and incubated overnight at $4^{\circ} \mathrm{C}$ with rotation. After confirmation by flow cytometry, NEEs were eluted from the beads using $300 \mu \mathrm{L}$ of Exosome Elution Buffer (System Biosciences, CA, United States; Catalog \#CSFLOWBASICA-1), and NEEs were used immediately or stored at $-80^{\circ} \mathrm{C}$ for miRNAs purification.

Flow Cytometry. Once the NEEs were captured and stabilized, the bead/antibody/exosome complex was coupled to a fluorescein isothiocyanate (FITC) fluorescent tag that specifically binds to exosomes (ExoFITC, Systems Biosciences; Cat \#CSFLOWBASICA-1) and subsequently analyzed by flow cytometry to confirm exosome capture (Fig. 4A). Briefly, the bead/antibody/exosome complex was washed three times with $1 X$ BWB and then incubated in $240 \mu \mathrm{L}$ of Exosome Stain Buffer and $10 \mu \mathrm{L}$ of EXO-FITC for 2 hours on ice with gentle flicking every 30 minutes. The stained complex was washed three times in 1X BWB and suspended in $500 \mu \mathrm{L} 1 \mathrm{X}$ BWB prior to flow cytometry loading. The flow cytometric data were acquired 
using a BD LSR II Special Order Flow Cytometer (BD Biosciences, San Jose, CA). Instrument performance was validated using BDTM Cytometer Setup and Tracking (CS\&T) beads (BD Biosciences, San Jose, CA). All data were analyzed using FACS DIVA 8.0 software (BD Biosciences). Debris and small particles were excluded by gating out events with low forward scatter. Figure 4B shows an example of successful exosome capture using the beads coated with CD171 antibodies specific for NEEs. Beads without exosomes were used as a negative control.

Western Blot. Enrichment of NEEs from TEs was confirmed by western blot (Fig. 4C). Protein concentrations in TEs and NEEs and exosome-depleted serum were determined using a Pierce ${ }^{\mathrm{TM}} \mathrm{BCA}$ protein assay (Thermo Fisher Scientific, USA, Catalog \# 23225). Samples were denatured directly in a $4 X$ Laemmli sample loading buffer and separated by SDS-PAGE using Mini PROTEAN ${ }^{\circ}$ TGX ${ }^{\text {TM }}$ precast gels (Bio-Rad, USA, Catalog \# 4561044). Following electrophoresis, gels were transferred unto polyvinylidene difluoride (PVDF) membranes using a Trans-Blot ${ }^{\circledR}$ Turbo transfer system (Bio-Rad, USA, Catalog \# 1704156). PVDF membranes were blocked with $5 \%$ non-fat milk powder in Tris-buffered saline containing 0.1\% Tween20 (Bio-Rad, USA, Catalog \# 1706435) and then incubated with primary mouse antibody against CD171 (1:1000, CD171 Monoclonal Antibody (eBio5G3 (5G3), eBioscience ${ }^{\mathrm{TM}}$, Catalog \# 13-171982) overnight at $4^{\circ} \mathrm{C}$. This was followed by incubation with a horseradish peroxidase-conjugated antimouse antibody (1:2000, Cell Signaling, Catalog \# 7076S) for 1 hour at room temperature. PVDF membranes were visualized by Clarity Max Western ECL Substrate (Bio-Rad, USA, Catalog \# 1705062) and imaged using ImageQuant LAS 4000 (GE Healthcare Bio-Science, Sweden). Image displayed in Fig. 4C was from the same gel, full-length gel is included in Supplemental Figure S1.

Nanoparticle analysis. Size and concentration of NEEs were determined using Nanoparticle Tracking Analysis system (NanoSight NS300, Malvern Panalytical Inc., Malvern, United Kingdom). Figure 4D showed that the majority of captured NEEs were in the exosome size range, with mean size of $175 \mathrm{~nm}$, and a standard deviation of $53 \mathrm{~nm}$; the average concentration of NEEs was approximately $1.6 \times 10^{8}$ particles per $\mathrm{mL}$.

NEEs MiR Purification. Purification was conducted using a Qiagen miRNeasy Micro Kit (QIAGEN, United States) according to the manufacturer's protocol. Small RNA concentration was measured using an Agilent Small RNA kit (Agilent, United States) on a Bioanalyzer 2100 instrument (Agilent, United States). MiR samples were stored at $-80^{\circ} \mathrm{C}$ until sequencing.

NEEs MiR sequencing and data processing. MiR samples were sent to the Oklahoma Medical Research Foundation (OMRF) Clinical Genomics Center for Next Generation Sequencing (NGS). MiR libraries were generated with a Qiagen QIAseq MiR library preparation kit and NGS was performed on an Illumina NextSeq HO SR75. Raw sequence FASTQ files received from OMRF were imported to Partek Flow software for data analysis. Adapters from 3 ' end were trimmed from the raw read after a quality check, and then aligned to the human genome hg38 using Bowtie alignment. Next, the aligned reads were quantified against the human miRbase mature microRNAs version 22 and reads from miR genes were normalized and scaled to reads per million for statistical data analysis. 
MID task. This task contained trials where participants saw a cue then a target, and the objective was to press a button as quickly as possible while the target was on the screen. Cues indicated the possible outcomes of a trial, with circle cues indicating a gain for hitting the target and square cues indicating a loss for missing the target. The magnitude of potential gain/loss was indicated by the position of a line on the cue and text showing the trial type $(-5 /-1 /-0 /+0 /+1 /+5)$. There were 90 trials ( 15 of each condition) split across two 568s runs. Target duration was calibrated based on a practice session completed before the scan and adjusted during scanning, so that on average participants hit on $60 \%$ of trials and earned $\$ 30$ for the task.

fMRI data were acquired during the MID task using two identical GE MR750 3T scanners using echoplanar imaging and the following parameters: 39 axial slices, $\mathrm{TR} / \mathrm{TE}=2000 / 27 \mathrm{~ms}$, FOV $/$ slice $=$ 240/2.9mm, $128 \times 128$ matrix. High-resolution structural images were obtained through a 3D axial T1weighted magnetization-prepared rapid acquisition with gradient echo (MP-RAGE) sequence (TR/TE = 5/2.0 $12 \mathrm{~ms}, \mathrm{FOV} /$ slice $=240 \times 192 / 0.9 \mathrm{~mm}, 186$ axial slices). $\mathrm{fMRI}$ preprocessing was done using Analysis of Functional Neuro Imaging (AFNI ${ }^{65}$ and consisted of: removal of the first three EPI volumes for signal stabilization, despiking, slice timing correction, co-registration to the anatomical image, motion correction via rigid-body alignment, and normalization to the Montreal Neurological Institute (MNI) standard space while resampling to $2 \times 2 \times 2$-mm voxels, and smoothing with a 4-mm full-width at halfmaximum filter. A general linear model was used to model the BOLD response during the anticipation phase of the MID with regressors for each of the 6 conditions $(-5 /-1 /-0 /+0 /+1 /+5)$ as well as the six motion parameters and four polynomial terms. Voxelwise beta coefficients representing percent signal change were taken to the group level.

Statistical analysis on NEEs MiR. Normalized MiR genes miR-27b-3p and miR-320b were log-transformed due to non-normality and used as the dependent variable in a repeated measure analysis of variance (ANOVA) with dose (placebo, ibuprofen $200 \mathrm{mg}$, ibuprofen $600 \mathrm{mg}$ ) as the within-subjects variable; paired $\mathrm{t}$-tests were employed to test mean differences between doses. Similar repeated ANOVA tests and paired t-tests were also estimated for other MiRs with enough reads for statistical analysis.

MID analyses. 3dLME ${ }^{66}$ was used to fit models with beta $~$ miR $^{\star}$ drug + visit $(\mathrm{T} 1 / \mathrm{T} 2 / \mathrm{T} 3)+$ condition for gains and losses separately. Random effects of subject and visit nested within subject were included. Four models were run, for gains/losses and miR-27b-3p/miR-320b. After fitting each model, smoothness of the residuals was estimated using 3dFWHMx. Then 3dClustSim was used to estimate the family-wise error rate (FWER) given voxel-wise and cluster-size thresholds. Results are reported with a voxel-wise threshold of $p<0.001$ and a FWER of $a<0.01$. Effect sizes are reported based on the same LME models run post-hoc on average percent signal change in significant clusters.

\section{Results}

\section{NEEs MiR Results}


There was a significant main effect of ibuprofen dose on miR-27b-3p ( $\left.F_{1,18}=5.38, p=0.015\right)$, miR-320b $\left(F_{1,18}=6.69, p=0.007\right)$, miR-23b-3p $\left(F_{1,18}=5.94, p=0.010\right)$, and miR-203a-3p $\left(F_{1,18}=3.88, p=0.040\right)$

(Fig. 5). Pairwise comparisons indicated that ibuprofen $600 \mathrm{mg}$ exhibited higher expression on: (1) miR$27 \mathrm{~b}-3 \mathrm{p}(p=0.014, d=0.746,95 \% \mathrm{Cl} 0.133-1.034), \operatorname{miR}-320 \mathrm{~b}(p=0.002, d=0.787,95 \% \mathrm{Cl} 0.269-1.006)$, $\operatorname{miR}-23 \mathrm{~b}-3 p(p=0.041, d=0.580,95 \% \mathrm{Cl} 0.026-1.072)$, and miR-203a-3p $(p=0.037, d=0.658,95 \% \mathrm{Cl}$ $0.045-1.251)$ than placebo; and (2) miR-27b-3p ( $p=0.046, d=0.469,95 \% \mathrm{Cl} 0.006-0.624)$, miR-320b ( $p$ $=0.048, d=0.445,95 \% \mathrm{Cl} 0.004-0.746)$ and miR-23b-3p ( $p=0.017, d=0.609,95 \% \mathrm{Cl} 0.092-0.836)$ than ibuprofen $200 \mathrm{mg}$. See Supplemental Table S1 for a list of other MiRs detected in NEEs.

\section{Neuroimaging Results}

Main effect of miR-27b-3p for MID loss anticipation in bilateral insula. Both the left (Center-of-mass $=41$, $-9,-15,156$ voxels, standardized $\beta=-0.36, p=0.001$ ) and right (Center-of-mass $=-41,-5,-11,62$ voxels, standardized $\beta=-0.0 .31, p=0.004$ ) insula showed a significant main effect of miR-27b-3p on activation during loss anticipation trials, with increased levels of miR-27b-3p related to decreased percent signal change (Fig. 6). Repeating the voxelwise analysis after removal of one outlier attenuated the effect, producing smaller clusters ( 75 voxels on the left, $a<0.01$ and 18 voxels on the right, $a>0.1$ ). There was no significant MiR by condition interaction.

Interaction between miR-27b-3p and condition for MID gain anticipation in bilateral putamen. Both the left (Center-of-mass $=31.5,6.1,-2.9,89$ voxels) and right (Center-of-mass $=-29.1,-2.7,1.2,80$ voxels) putamen showed a significant interaction between miR-27b-3p and condition on activation during the MID task during gain anticipation (Fig. 7). Post-hoc analyses showed the negative relationship between miR-27b-3p and activation in the putamen during placebo (standardized $\beta=-0.21, p=0.02$ ) was attenuated by both $200 \mathrm{mg}$ (interaction standardized $\beta=0.32, p=6.7^{*} 10^{-5}$ ) and $600 \mathrm{mg}$ (interaction standardized $\beta=0.16, p=0.02$ ) of ibuprofen.

Other Effects of miR-27b-3p and miR-320b. Several other regions showing either a main effect of miR or a miR by condition interaction are listed in Supplemental Tables S2 through S5.

\section{Discussion}

This investigation examined the effect of an acute administration of ibuprofen on miRs modulating inflammation-related regulatory processes identified from NEEs with RNA seq analysis, and their effects on CNS function as examined by fMRI during anticipation of rewards and losses. There were three main results. First, ibuprofen dose-dependently increased miR-27b-3p, miR-320b, miR-23b-3p and miR-203a-3p, which are important modulators of various aspects of brain function. Second, higher miR-27b-3p expressions were linked to lower bilateral ventral insula responses within the context of loss anticipation. Third, ibuprofen moderated the effect of miR-27b-3p on the anticipation of gains in bilateral putamen such that both the $200 \mathrm{mg}$ and $600 \mathrm{mg}$ doses elicited positive correlations between miR-27b-3p and putamen activation during anticipation of gains, wherein the placebo dose elicited a negative correlation. 
Taken together, these findings support the notion that ibuprofen's effects in the brain are related to modulatory miRs that can blunt the relationship between the degree of inflammation and reward-related processing.

These findings have several implications for the understanding of how anti-inflammatory drugs may affect the brain and how these brain changes may contribute to altered processing of positive or negative-valenced stimuli. The MID task has been used extensively to examine reward and loss related processing in individuals with mood or anxiety disorders ${ }^{50}$. Specifically, individuals with depression show an attenuation of reward-related anticipation during an episode ${ }^{51}$ but not when remitted ${ }^{52}$ and may normalize after treatment ${ }^{53}$. Moreover, increased levels of peripheral inflammatory markers are associated with relatively lower reward-related activation ${ }^{54}$, which is consistent with the view that inflammatory cytokines adversely affect positive valence processing ${ }^{49}$. The findings from this study suggest that one possible molecular mechanism by which inflammation may affect reward-related processing is via the signaling of inflammation-modulating miRs contained in NEEs. However, the current results further suggest that this effect is not necessarily limited to reward related processes but extends also to the degree to which the brain processes loss events. Moreover, the imaging findings support the notion that these effects are not non-specific but involve brain structures that are important for gain or loss processing, specifically, insula and putamen that are robustly activated for both gain and loss anticipations during the MID task ${ }^{55}$. One particular target with an important role on brain and inflammation are PPARs, anti-inflammatory transcription factors that affect attenuating degenerative processes in the brain and are associated with control of anti-inflammatory mechanisms ${ }^{56}$. Several human studies have been conducted to examine the effect of PPARy agonists on mood 57 , including a double-blind placebo-controlled study, which showed that pioglitazone, a PPARy agonist improved mood in $\mathrm{MDD}^{58}$. The ibuprofen-induced attenuation of the relationship between individuals with relatively higher levels of miRs targeting the PPAR system and brain activation during anticipation of gains or losses may provide some mechanistic explanation of how anti-inflammatory agents might partially be able to rescue attenuated brain activation in the presence of high levels of inflammation.

However, the effects of miRs may not necessarily be limited to inflammatory-related processes. As shown in Figs. 1 and 2, both miR-27b-3p, miR-320b have multiple targets that can have modulatory effects on brain function. This is consistent with findings from case-control studies in anxiety or depression showing evidence for an increase in miRs including miR-27b-3 ${ }^{59}$ and miR-320 ${ }^{34}$, which has been more closely related to metabolic dysfunction ${ }^{19,20}$. For example, global upregulation of miR-320 has been associated with impaired gluconeogenesis, lipid metabolism, and relatively higher expression of inflammation markers ${ }^{60}$. The integration of functional neuroimaging studies with molecular assessments using miRs obtained from NEEs open the possibility of identifying novel, targetable disease-modifying processes in mood or anxiety disorder. Disease modifying processes can be based on circuits, behavior, or other units of analysis, which - when modulated - change the risk for, severity of, or recurrence of a disease such as mood and anxiety disorders. The use of molecular tools enables one to determine whether systems are affected that can be targeted by a pharmacological intervention. The findings from 
this study provide evidence that identifiable, neuronally exosomal-enriched regulatory miRs are promising targets for modulating reward and loss related processing in both subcortical and cortical brain regions.

This study has several limitations. First, the participants were healthy volunteers with no symptoms of anxiety or depression. Therefore, further studies are needed to assess the relationship between the molecular effects of ibuprofen and changes in self-reported symptoms. Second, although we observed acute changes in NEE miRs associated with inflammation-related regulatory processes, it is not clear that these changes are sufficient to be therapeutic targets or whether these changes can be maintained with chronic dosing. Third, ibuprofen had limited direct effects on reward or loss related processing in this study; thus, future investigation may focus on other anti-inflammatory modulators that may have more profound effects on brain processing. Fourth, we were unable to assess other contents within the NEEs, which may provide more direct evidence for pathways involved in the ibuprofen-mediated effects in the brain. Nevertheless, this study provides sufficient proof-of-concept evidence and delineates a limited number of molecular processes that affect the brain's response to rewards or losses.

\section{Conclusions}

The results from this study are consistent with the hypothesis that miR in NEE could be important messaging molecules that are altered by anti-inflammatory drugs and alter the processing of positive or negative valenced information. In this case, ibuprofen seems to attenuate the inverse relationship between inflammatory processes and reward-related activation in healthy volunteers.

\section{Declarations}

\section{Funding}

This work was supported by The William K. Warren Foundation, and the National Institute of General Medical Sciences Center Grant Award (1P20GM121312).

\section{Acknowledgements}

The authors wish to thank Ashlee Rempel and Brenda Davis at the Integrative Immunology Center (IIC), School of Community Medicine, The University of Oklahoma, Tulsa, OK for their assistance in the data collection. Special thanks to Dr. Chibing Tan from IIC for his help with the flow cytometry experiments. The authors wish to thank Dr. Bethany N. Hannafon from Department of Obstetrics and Gynecology, University of Oklahoma Health Sciences Center for her help with western blot. The nanoparticle analysis was conducted in Small Animal Bioluminescence Imaging Core, Stephenson Cancer Center, University of Oklahoma Health Sciences Center, Oklahoma City, OK. Special thanks to Dr. Akhil Srivastava for his help with the nanoparticle tracking analysis.

\section{Author contributions}


K.B. contributed to research design, data collection, data analysis, and manuscript writing and preparation; L.K.F. contributed to manuscript writing and preparation, creation of figures 1, 2 and 3; R.K. contributed to imaging data collection and analysis, manuscript writing and preparation; J.L.S. contributed to manuscript writing and preparation; A.M.A. contributed to exosome western blot analysis, and manuscript writing; R.R. contributed to exosome nanoparticle analysis, review and editing of the manuscript; J.B.S. contributed to critical manuscript review; T.K.T. contributed to study design, exosome flow cytometry analysis, manuscript writing and review; V.B.R. contributed to exosome study design, supervision of exosome data collection and analysis; M.P.P. contributed to study design, supervision of data collection and analysis, manuscript writing and preparation, and critical review of the manuscript. All authors reviewed the manuscript.

\section{Competing interests}

None of the authors have any financial or personal conflict of interest.

\section{References}

1. Savitz, J. \& Harrison, N. A. Interoception and Inflammation in Psychiatric Disorders. Biol Psychiatry Cogn Neurosci Neuroimaging, 3, 514-524 https://doi.org/10.1016/j.bpsc.2017.12.011 (2018).

2. Beurel, E., Toups, M. \& Nemeroff, C. B. The Bidirectional Relationship of Depression and Inflammation: Double Trouble., https://doi.org/10.1016/j.neuron.2020.06.002 (2020).

3. Halaris, A. Inflammation-Associated Co-morbidity Between Depression and Cardiovascular Disease.Curr Top Behav Neurosci31,45-70, doi:10.1007/7854_2016_28

4. 1007/7854_2016_28. (2017).

5. Walker, A. K., Kavelaars, A., Heijnen, C. J. \& Dantzer, R. Neuroinflammation and comorbidity of pain and depression. Pharmacol Rev, 66, 80-101 https://doi.org/10.1124/pr.113.008144 (2014).

6. Khandaker, G. M., Pearson, R. M., Zammit, S., Lewis, G. \& Jones, P. B. Association of serum interleukin 6 and C-reactive protein in childhood with depression and psychosis in young adult life: a populationbased longitudinal study. JAMA Psychiatry, 71, 1121-1128 https://doi.org/10.1001/jamapsychiatry.2014.1332 (2014).

7. Dowlati, Y. et al. A meta-analysis of cytokines in major depression.Biol Psychiatry67,446-457, doi:10.1016/j.biopsych.2009.09.033

8. 1016/j.biopsych.2009.09.033. Epub 2009 Dec 16. (2010).

9. Zorrilla, E. P. et al. The relationship of depression and stressors to immunological assays: a metaanalytic review. Brain Behav Immun, 15, 199-226 https://doi.org/10.1006/brbi.2000.0597 (2001).

10. Treadway, M. T. et al. Association Between Interleukin-6 and Striatal Prediction-Error Signals Following Acute Stress in Healthy Female Participants. Biol Psychiatry, 82, 570-577 https://doi.org/10.1016/j.biopsych.2017.02.1183 (2017). 
11. Burrows, K. et al. Elevated peripheral inflammation is associated with attenuated striatal reward anticipation in major depressive disorder. Brain Behav Immun, https://doi.org/10.1016/j.bbi.2021.01.016 (2021).

12. Kohler, O., Krogh, J., Mors, O. \& Benros, M. E. Inflammation in Depression and the Potential for AntiInflammatory Treatment. Curr Neuropharmacol, 14, 732-742 https://doi.org/10.2174/1570159x14666151208113700 (2016).

13. Rainsford, K. D. Ibuprofen: from invention to an OTC therapeutic mainstay. Int J Clin Pract Suppl, 920 https://doi.org/10.1111/ijcp.12055 (2013).

14. Matos, P., Jordan, P. \& Beyond COX-inhibition: 'side-effects' of ibuprofen on neoplastic development and progression. Curr Pharm Des, 21, 2978-2982 https://doi.org/10.2174/1381612821666150514104608 (2015).

15. Dill, J. et al. A molecular mechanism for ibuprofen-mediated RhoA inhibition in neurons. J Neurosci, 30, 963-972 https://doi.org/10.1523/JNEUROSCI.5045-09.2010 (2010).

16. Roloff, F. et al. Enhanced neurite outgrowth of human model (NT2) neurons by small-molecule inhibitors of Rho/ROCK signaling. PLoS One, 10, e0118536 https://doi.org/10.1371/journal.pone.0118536 (2015).

17. Delli Pizzi, S. et al. Pharmacological functional MRI assessment of the effect of ibuprofen-arginine in painful conditions. Int J Immunopathol Pharmacol, 23, 927-935 https://doi.org/10.1177/039463201002300329 (2010).

18. Wanigasekera, V., Mezue, M., Andersson, J., Kong, Y. \& Tracey, I. Disambiguating Pharmacodynamic Efficacy from Behavior with Neuroimaging: Implications for Analgesic Drug Development., 124, 159168 https://doi.org/10.1097/ALN.0000000000000924 (2016).

19. Karthikeyan, A. et al. Key Players in Microglia and Astrocyte Mediated Inflammation in CNS Pathologies. Curr Med Chem, 23, 3528-3546 https://doi.org/10.2174/0929867323666160814001040 (2016).

20. Ding, L. et al. Promising therapeutic role of miR-27b in tumor. Tumour Biol, 39, 1010428317691657 https://doi.org/10.1177/1010428317691657 (2017).

21. Vickers, K. C. et al. MicroRNA-27b is a regulatory hub in lipid metabolism and is altered in dyslipidemia., 57, 533-542 https://doi.org/10.1002/hep.25846 (2013).

22. Yu, J. et al. MiR-27b-3p Regulation in Browning of Human Visceral Adipose Related to Central Obesity. Obesity (Silver Spring), 26, 387-396 https://doi.org/10.1002/oby.22104 (2018).

23. Karbiener, M. et al. microRNA miR-27b impairs human adipocyte differentiation and targets PPARgamma. Biochem Biophys Res Commun, 390, 247-251 https://doi.org/10.1016/j.bbrc.2009.09.098 (2009).

24. Wang, L. et al. Hsa-miR-27b is up-regulated in cytomegalovirus-infected human glioma cells, targets engrailed-2 and inhibits its expression. Exp Biol Med (Maywood), 242, 1227-1233 https://doi.org/10.1177/1535370217699535 (2017). 
25. Patel, S. A. et al. Interleukin-6 mediated upregulation of CYP1B1 and CYP2E1 in colorectal cancer involves DNA methylation, miR27b and STAT3. Br J Cancer, 111, 2287-2296 https://doi.org/10.1038/bjc.2014.540 (2014).

26. Lee, J. J., Drakaki, A., Iliopoulos, D. \& Struhl, K. MiR-27b targets PPARgamma to inhibit growth, tumor progression and the inflammatory response in neuroblastoma cells., $31,3818-3825$ https://doi.org/10.1038/onc.2011.543 (2012).

27. Boon, R. A., Hergenreider, E. \& Dimmeler, S. Atheroprotective mechanisms of shear stress-regulated microRNAs. Thromb Haemost, 108, 616-620 https://doi.org/10.1160/TH12-07-0491 (2012).

28. Dias, S., Hemmings, S., Muller, C., Louw, J. \& Pheiffer, C. MicroRNA Expression Varies according to Glucose Tolerance, Measurement Platform, and Biological Source. Biomed Res Int 2017, 1080157, doi:10.1155/2017/1080157 (2017).

29. Kida, K. et al. PPARalpha is regulated by miR-21 and miR-27b in human liver. Pharm Res, 28, 24672476 https://doi.org/10.1007/s11095-011-0473-y (2011).

30. Al-Eitan, L. N., Alghamdi, M. A., Tarkhan, A. H. \& Al-Qarqaz, F. A. Gene Expression Profiling of MicroRNAs in HPV-Induced Warts and Normal Skin. Biomolecules, $\mathbf{9}$, https://doi.org/10.3390/biom9120757 (2019).

31. Poon, V. Y., Gu, M., Ji, F., VanDongen, A. M. \& Fivaz, M. miR-27b shapes the presynaptic transcriptome and influences neurotransmission by silencing the polycomb group protein Bmi1. BMC Genomics, 17, 777 https://doi.org/10.1186/s12864-016-3139-7 (2016).

32. Giorgi Silveira, R. et al. MicroRNAs expressed in neuronal differentiation and their associated pathways: Systematic review and bioinformatics analysis. Brain Res Bul/157,140-148, doi:10.1016/j.brainresbull.2020.01.009

33. 1016/j.brainresbull.2020.01.009. Epub 2020 Jan 13. (2020).

34. Du, H., Zhao, Y., Yin, Z., Wang, D. W. \& Chen, C. The role of miR-320 in glucose and lipid metabolism disorder-associated diseases. Int J Biol Sci, 17, 402-416 https://doi.org/10.7150/ijbs.53419 (2021).

35. Pierdomenico, M. et al. NOD2 Is Regulated By Mir-320 in Physiological Conditions but this Control Is Altered in Inflamed Tissues of Patients with Inflammatory Bowel Disease. Inflamm Bowel Dis, 22, 315-326 https://doi.org/10.1097/MIB.0000000000000659 (2016).

36. Liu, L. \& Li, X. Downregulation of miR-320 Alleviates Endoplasmic Reticulum Stress and Inflammatory Response in 3T3-L1 Adipocytes. Exp Clin Endocrinol Diabetes, 129, 131-137 https://doi.org/10.1055/a-1012-8420 (2021).

37. Fiori, L. M. et al. miR-323a regulates ERBB4 and is involved in depression.Mol Psychiatry, doi:10.1038/s41380-020-00953-7

38. 1038/s41380-020-00953-7. (2020).

39. Rainville, P., Duncan, G. H., Price, D. D., Carrier, B. \& Bushnell, M. C. Pain affect encoded in human anterior cingulate but not somatosensory cortex., 277, 968-971 https://doi.org/10.1126/science.277.5328.968 (1997). 
40. Shelton, L., Becerra, L. \& Borsook, D. Unmasking the mysteries of the habenula in pain and analgesia. Prog Neurobiol, 96, 208-219 https://doi.org/10.1016/j.pneurobio.2012.01.004 (2012).

41. Lieb, V. et al. Serum levels of miR-320 family members are associated with clinical parameters and diagnosis in prostate cancer patients. Oncotarget, 9, 10402-10416 https://doi.org/10.18632/oncotarget.23781 (2018).

42. Paschon, V. et al. Interplay Between Exosomes, microRNAs and Toll-Like Receptors in Brain Disorders. Mol Neurobiol, 53, 2016-2028 https://doi.org/10.1007/s12035-015-9142-1 (2016).

43. Paolicelli, R. C., Bergamini, G. \& Rajendran, L. Cell-to-cell Communication by Extracellular Vesicles: Focus on Microglia., 405, 148-157 https://doi.org/10.1016/j.neuroscience.2018.04.003 (2019).

44. Hu, G., Drescher, K. M. \& Chen, X. M. Exosomal miRNAs: Biological Properties and Therapeutic Potential. Front Genet, 3, 56 https://doi.org/10.3389/fgene.2012.00056 (2012).

45. Cheng, L., Sharples, R. A., Scicluna, B. J. \& Hill, A. F. Exosomes provide a protective and enriched source of miRNA for biomarker profiling compared to intracellular and cell-free blood. $J$ Extracell Vesicles, 3, https://doi.org/10.3402/jev.v3.23743 (2014).

46. Zhou, Y. et al. Exosomes Transfer Among Different Species Cells and Mediating miRNAs Delivery.J Cell Biochem118,4267-4274, doi:10.1002/jcb.26077

47. 1002/jcb.26077. Epub 2017 May 25. (2017).

48. Yang, Y. et al. Inflammation leads to distinct populations of extracellular vesicles from microglia. Journal of Neuroinflammation, 15, 168 https://doi.org/10.1186/s12974-018-1204-7 (2018).

49. Grimaldi, A. et al. Microglia-Derived Microvesicles Affect Microglia Phenotype in Glioma. Frontiers in Cellular Neuroscience, 13, https://doi.org/10.3389/fncel.2019.00041 (2019).

50. Murgoci, A. N. et al. Brain-Cortex Microglia-Derived Exosomes: Nanoparticles for Glioma Therapy., 19, 1205-1214 https://doi.org/10.1002/cphc.201701198 (2018).

51. Brites, D. \& Fernandes, A. Neuroinflammation and Depression: Microglia Activation, Extracellular Microvesicles and microRNA Dysregulation.Front Cell Neurosci9,476, doi:10.3389/fncel.2015.00476

52. 3389/fncel.2015.00476. eCollection 2015. (2015).

53. Knutson, B., Adams, C. M., Fong, G. W. \& Hommer, D. Anticipation of increasing monetary reward selectively recruits nucleus accumbens. $J$ Neurosci 21, Rc159, doi:10.1523/JNEUROSCI.21-16j0002.2001 (2001).

54. Goldsmith, D. R. \& Rapaport, M. H. Inflammation and Negative Symptoms of Schizophrenia: Implications for Reward Processing and Motivational Deficits. Front Psychiatry, 11, 46 https://doi.org/10.3389/fpsyt.2020.00046 (2020).

55. Felger, J. C. Imaging the Role of Inflammation in Mood and Anxiety-related Disorders. Curr Neuropharmacol, 16, 533-558 https://doi.org/10.2174/1570159X15666171123201142 (2018).

56. Rizvi, S. J., Pizzagalli, D. A., Sproule, B. A. \& Kennedy, S. H. Assessing anhedonia in depression: Potentials and pitfalls. Neurosci Biobehav Rev, 65, 21-35 https://doi.org/10.1016/j.neubiorev.2016.03.004 (2016). 
57. Pizzagalli, D. A. et al. Reduced caudate and nucleus accumbens response to rewards in unmedicated individuals with major depressive disorder. Am J Psychiatry, 166, 702-710 https://doi.org/10.1176/appi.ajp.2008.08081201 (2009).

58. Dichter, G. S., Kozink, R. V., McClernon, F. J. \& Smoski, M. J. Remitted major depression is characterized by reward network hyperactivation during reward anticipation and hypoactivation during reward outcomes. J Affect Disord, 136, 1126-1134 https://doi.org/10.1016/j.jad.2011.09.048 (2012).

59. Dunlop, K. et al. Clinical, behavioral, and neural measures of reward processing correlate with escitalopram response in depression: a Canadian Biomarker Integration Network in Depression (CANBIND-1) Report. Neuropsychopharmacology, 45, 1390-1397 https://doi.org/10.1038/s41386-0200688-x (2020).

60. Burrows, K. et al. Elevated peripheral inflammation is associated with attenuated striatal reward anticipation in major depressive disorder. Brain Behav Immun, 93, 214-225 https://doi.org/10.1016/j.bbi.2021.01.016 (2021).

61. Wilson, R. P. et al. The Neural Substrate of Reward Anticipation in Health: A Meta-Analysis of fMRI Findings in the Monetary Incentive Delay Task. Neuropsychol Rev, 28, 496-506 https://doi.org/10.1007/s11065-018-9385-5 (2018).

62. Villapol, S. Roles of Peroxisome Proliferator-Activated Receptor Gamma on Brain and Peripheral Inflammation. Cell Mol Neurobiol, 38, 121-132 https://doi.org/10.1007/s10571-017-0554-5 (2018).

63. Kashani, L. et al. Does pioglitazone improve depression through insulin-sensitization? Results of a randomized double-blind metformin-controlled trial in patients with polycystic ovarian syndrome and comorbid depression., 38, 767-776 https://doi.org/10.1016/j.psyneuen.2012.08.010 (2013).

64. Sepanjnia, K., Modabbernia, A., Ashrafi, M., Modabbernia, M. J. \& Akhondzadeh, S. Pioglitazone adjunctive therapy for moderate-to-severe major depressive disorder: randomized double-blind placebo-controlled trial. Neuropsychopharmacology, 37, 2093-2100 https://doi.org/10.1038/npp.2012.58 (2012).

65. Zastrozhin, M. S. et al. Effects of plasma concentration of micro-RNA Mir-27b and CYP3A4*22 on equilibrium concentration of alprazolam in patients with anxiety disorders comorbid with alcohol use disorder., 739, 144513 https://doi.org/10.1016/j.gene.2020.144513 (2020).

66. Wei, G. et al. miR-320 mediates diabetes amelioration after duodenal-jejunal bypass via targeting adipoR1. Surg Obes Relat Dis, 14, 960-971 https://doi.org/10.1016/j.soard.2018.03.007 (2018).

67. Mustapic, M. et al. Plasma Extracellular Vesicles Enriched for Neuronal Origin: A Potential Window into Brain Pathologic Processes.Front Neurosci11,278, doi:10.3389/fnins.2017.00278

68. 3389/fnins.2017.00278. eCollection 2017. (2017).

69. Winston, C. N. et al. Assessing Neuronal and Astrocyte Derived Exosomes From Individuals With Mild Traumatic Brain Injury for Markers of Neurodegeneration and Cytotoxic Activity. Frontiers in Neuroscience, 13, https://doi.org/10.3389/fnins.2019.01005 (2019). 
70. Pulliam, L., Sun, B., Mustapic, M., Chawla, S. \& Kapogiannis, D. Plasma neuronal exosomes serve as biomarkers of cognitive impairment in HIV infection and Alzheimer's disease. J Neurovirol, 25, 702709 https://doi.org/10.1007/s13365-018-0695-4 (2019).

71. Sun, B., Dalvi, P., Abadjian, L., Tang, N. \& Pulliam, L. Blood neuron-derived exosomes as biomarkers of cognitive impairment in HIV. AIDS 31, F9-F17, doi:10.1097/QAD.0000000000001595

72. 1097/QAD.0000000000001595. (2017).

73. Cox, R. W. AFNI: software for analysis and visualization of functional magnetic resonance neuroimages. Comput Biomed Res, 29, 162-173 https://doi.org/10.1006/cbmr.1996.0014 (1996).

74. Chen, G., Saad, Z. S., Britton, J. C., Pine, D. S. \& Cox, R. W. Linear mixed-effects modeling approach to FMRI group analysis., 73, 176-190 https://doi.org/10.1016/j.neuroimage.2013.01.047 (2013).

\section{Figures}

A.

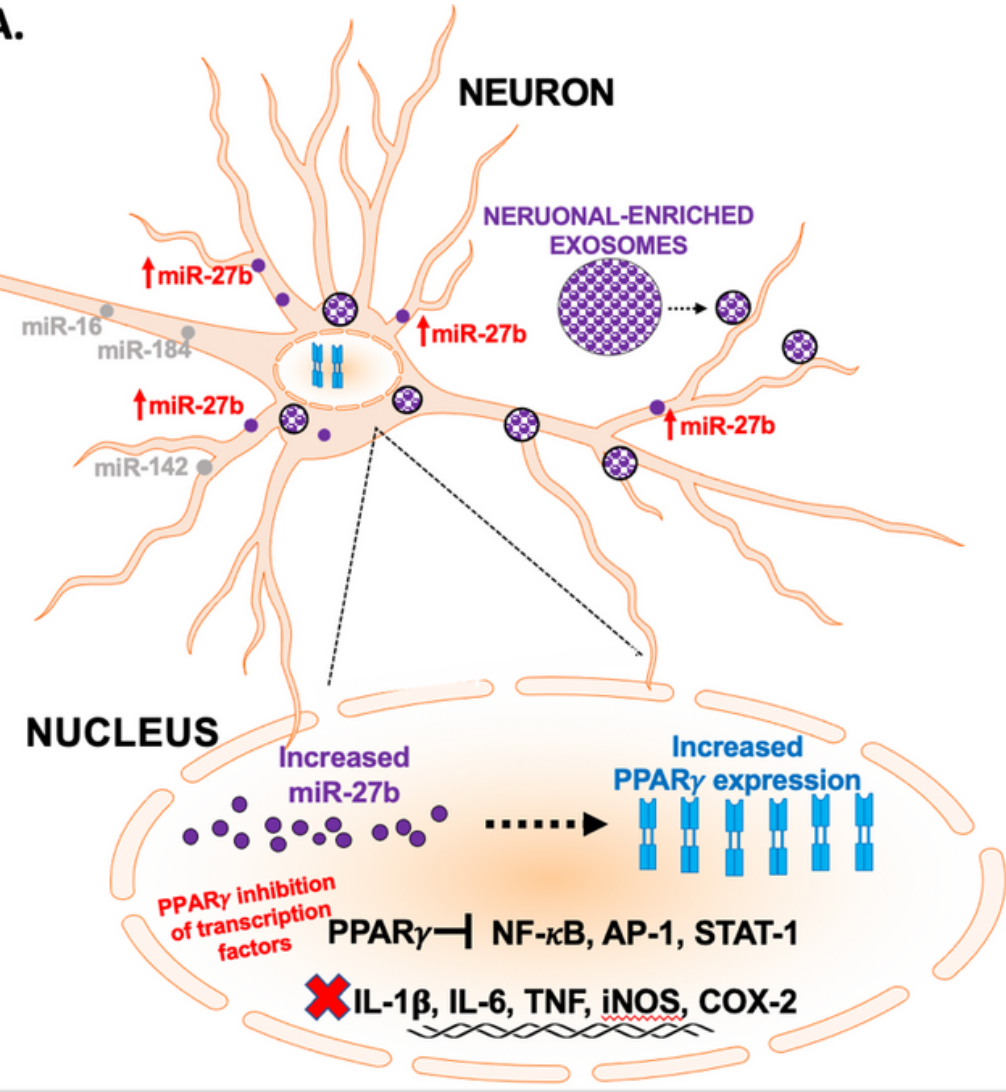

B.

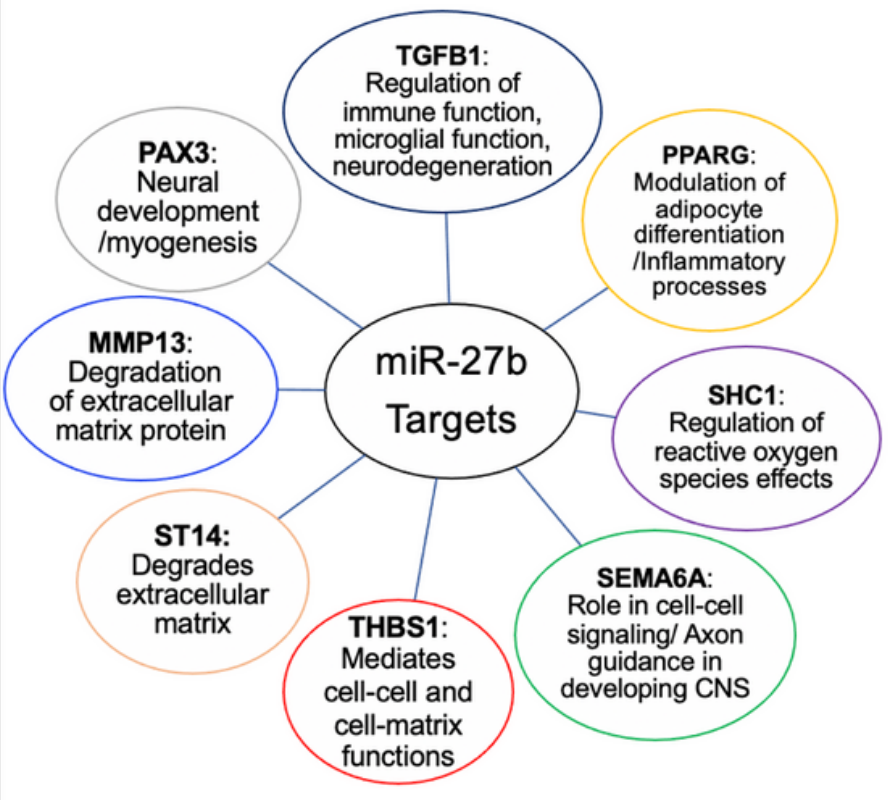

\section{Figure 1}

Regulatory role and targets of miR-27b. Neuronally-enriched exosomes (NEEs) are shown to express miRNAs for intracellular communication and aberrant expression in various health conditions. A) Significant increases in NEE mir-27b can lead to regulation of several targets including increased expression of PPAR, which inhibits the expression of several transcription factors, such as NF-B and AP-1, leading to the attenuation of proinflammatory mediators including IL-6 and TNF. B) Other miR-27b gene targets include PAX3, TGFB1, PPARG, SHC1, MMP13, ST14, THBS1, and SEMA6A that mediate and 
regulate several functions including neuronal development, inflammatory processes, degradation of extracellular matrix, and cell-cell functions. Abbreviations- PPAR: Peroxisome Proliferator-activated receptor gamma; NF-kB: Nuclear Factor kappa-light-chain-enhancer of activated B cells; AP-1: Activator Protein 1; STAT-1: Signal Transducer and activator of transcription 1; IL-1B: interleukin-1 beta; IL-6: interleukin-6; TNF: tumor necrosis factor; iNOS: inducible nitric oxide synthase; COX-2: cyclooxygenase 2; TGFB1: transforming growth factor beta 1; SHC1: Src homology 2 domain containing transforming protein 1; SEMA6A: Semaphorin 6A; THBS1: Thrombospondin 1 ; ST14: Suppressor of Tumorigenicity 1; MMP13: Matrix Metallopeptidase 13; PAX3: Paired Box 3.

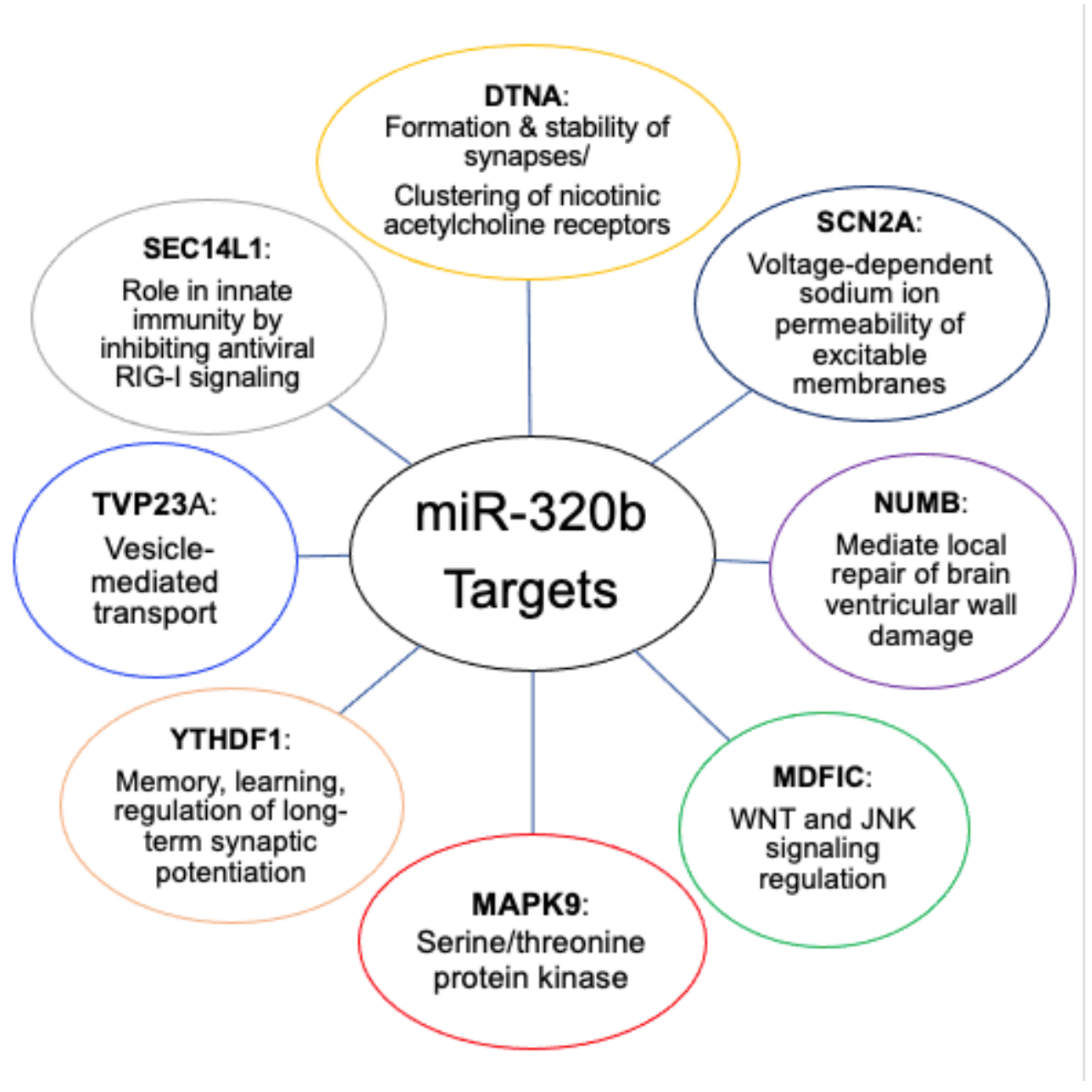

Figure 2

miR-320b gene targets and functions. miR-320b acts on various gene targets including DTNA, SCN2A, NUMB, MDFIC, MAPK9, YTHDF1, TVP23A, and SEC14L1. These genes mediate several functions including formation and stability of synapses, local repair of brain ventricular wall damage, WNT and JNK signaling, serine/threonine protein kinase activation, memory, learning, and long-term synaptic potentiation, vesicle-mediated transport and innate immunity through RIG-I signaling. Abbreviations: DTNA: Dystrobrevin Alpha; SCN2A: Sodium Voltage-gated Channel Alpha Subunit 2; NUMB: NUMB endocytic adaptor protein; MDFIC: MyoD Family Inhibitor Domain Containing Protein; MAPK9: MitogenActivated Protein Kinase 9; YTHDF1: YTH N6-Methyladenosine RNA Binding Protein 1; TVP23A: Trans- 
Golgi Network Vesicle Protein 23 Homolog A; SEC14L1: SEC14 Like Lipid Binding 1; WNT: winglessrelated intergration site; and JNK: c-jun N-terminal kinase.

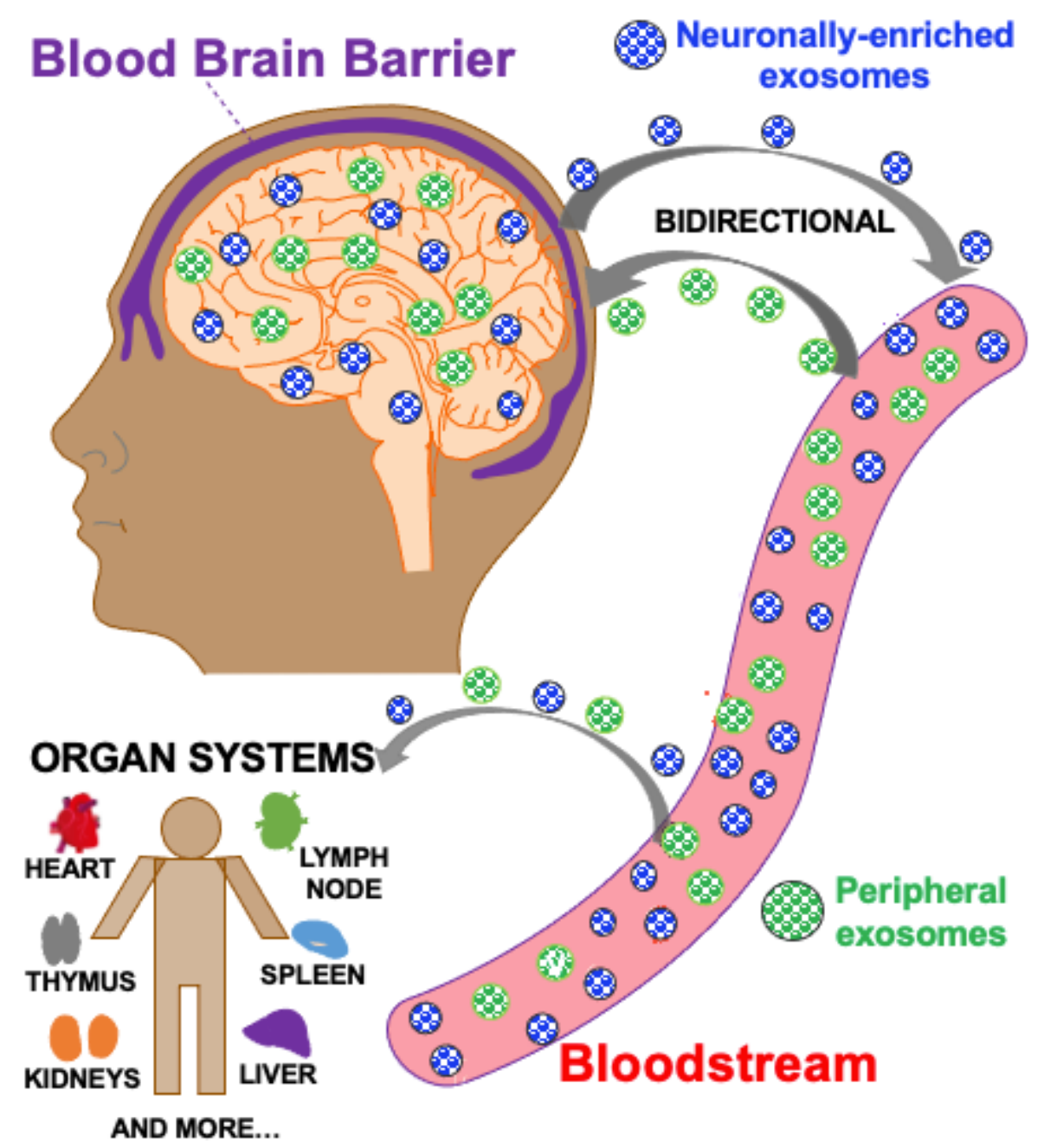

Figure 3

Neuronally-enriched exosomal pathway. NEEs (blue) are released from neurons in the central nervous system (CNS) and cross the blood brain barrier (BBB) into the bloodstream. This allows for NEE capture from platforms including serum and plasma. Peripheral exosomes (purple) can also cross the BBB into the CNS to mediate a bidirectional pathway. Exosomes in the bloodstream also travel to different organ systems in the body including the heart, liver, kidneys, etc. 

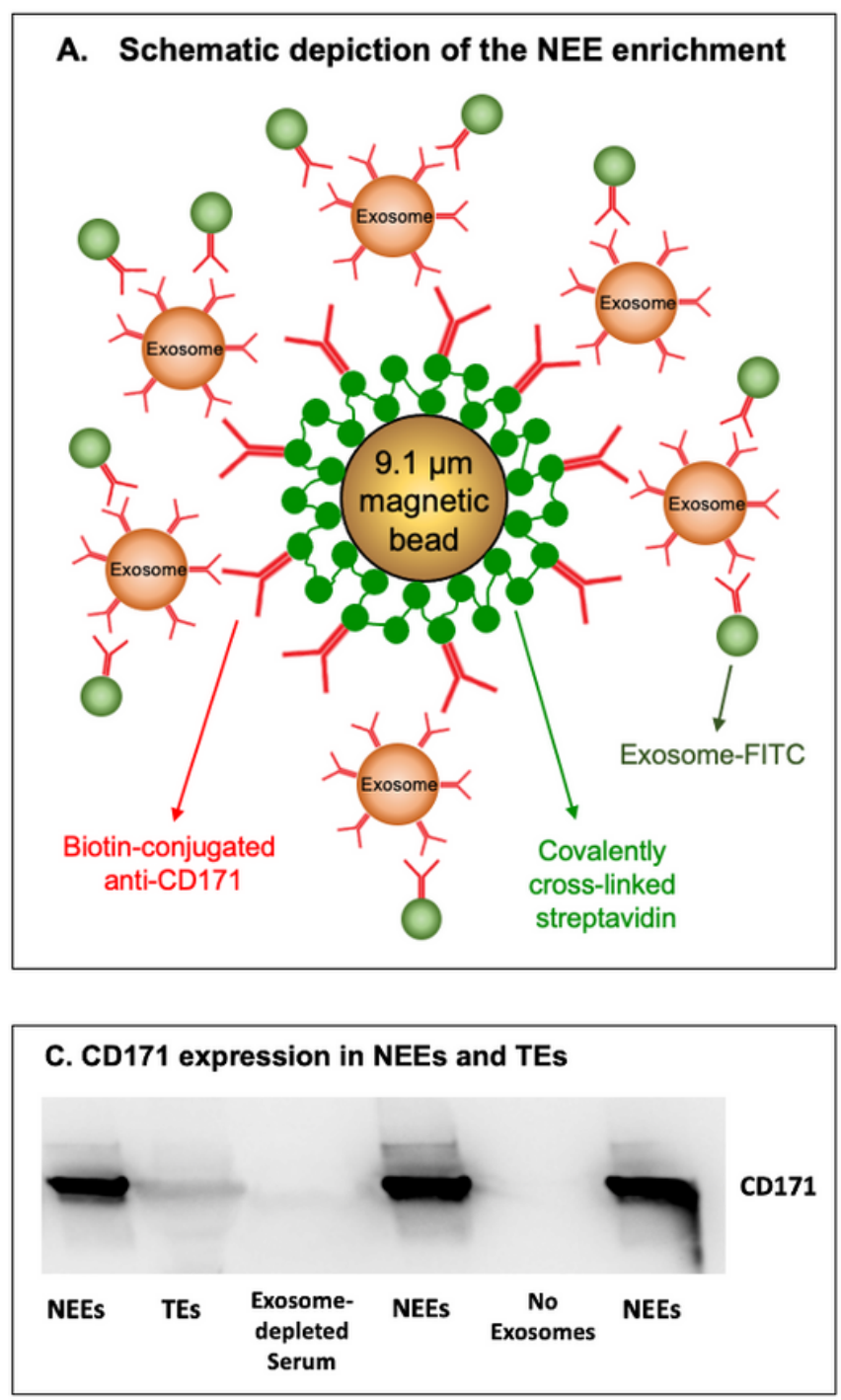

B. FACS results of NEEs and Negative Control

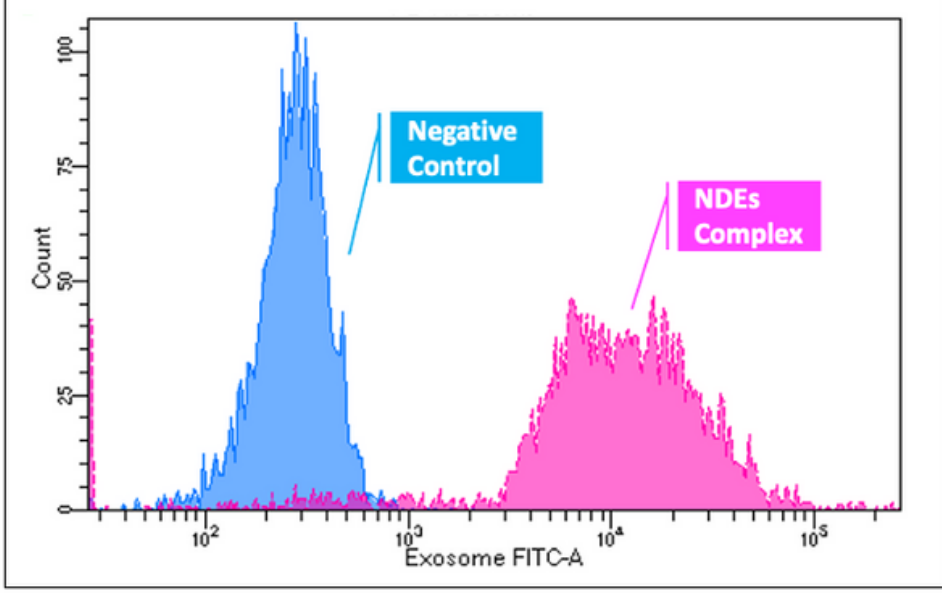

\section{Size and concentration of NEEs}

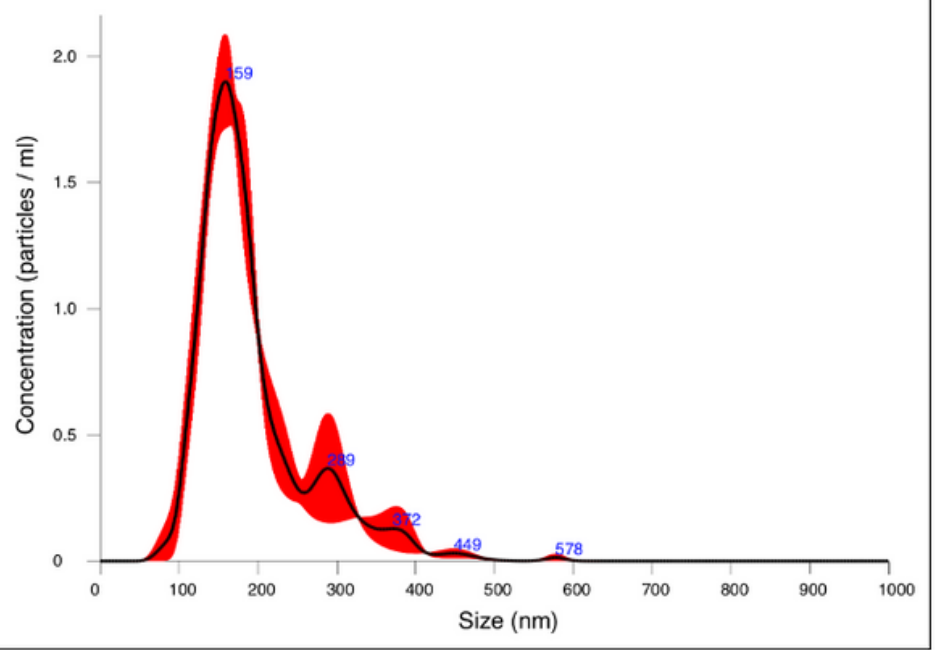

\section{Figure 4}

Neuronally-enriched exosomes (NEEs) A. Schematic depiction of NEE enrichment. B. Fluorescence activated cell sorting (FACS) results of NEEs and negative control (No exosomes). C. Western blot analysis of NEEs, total exosomes (TEs), exosome-depleted serum, and negative controls (No exosomes) with anti-CD171 antibody marker, image cropped was from the same gel. D. Size and concentration analysis of NEEs using Nanoparticle Tracking Analysis System. 
miR-27b-3p

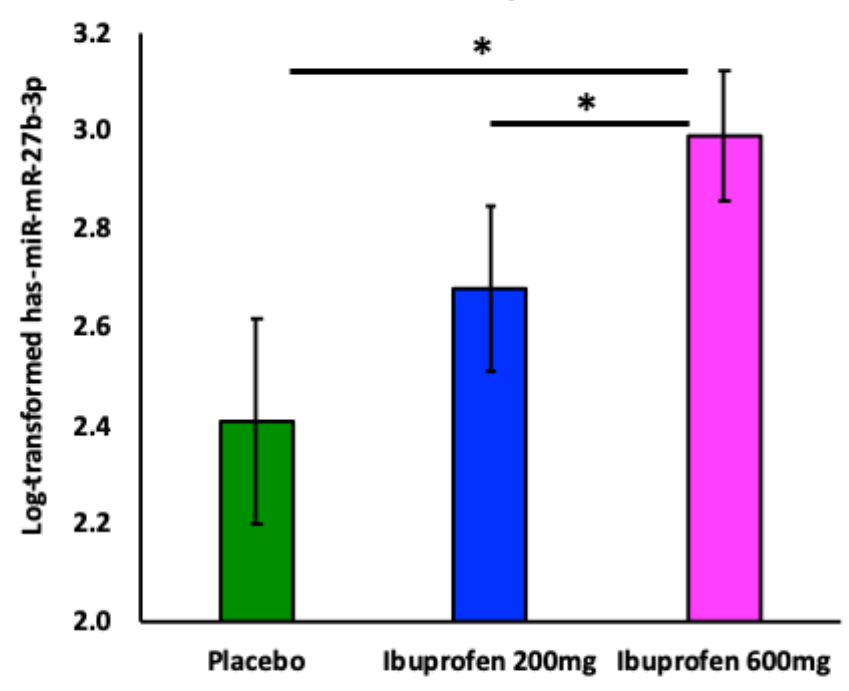

miR-23b-3p

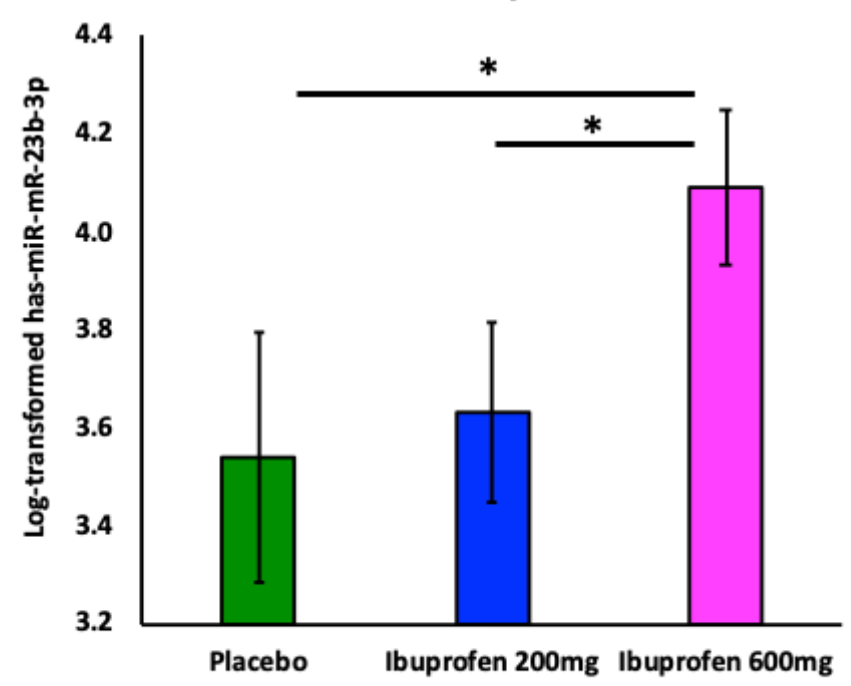

miR-320b

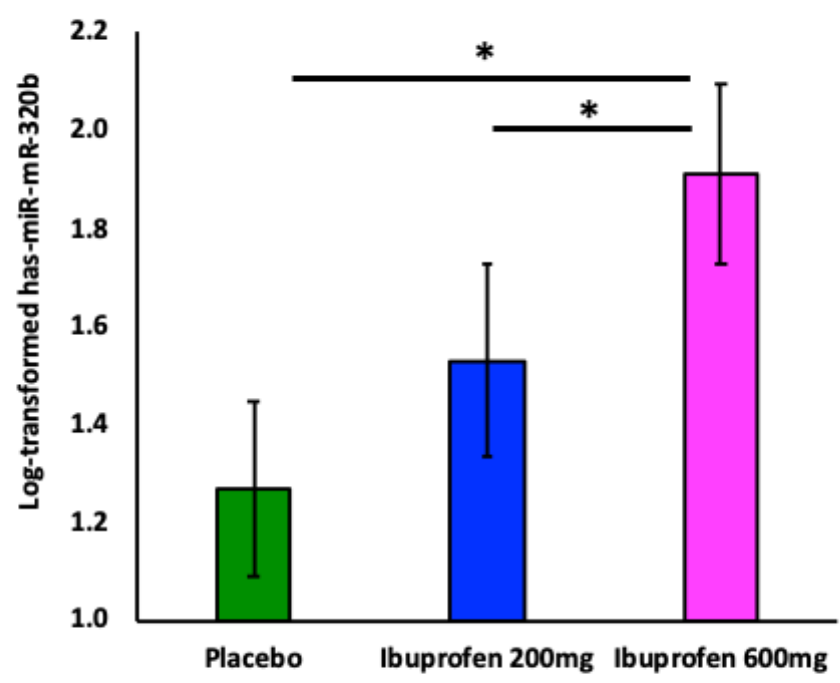

miR-203a-3p

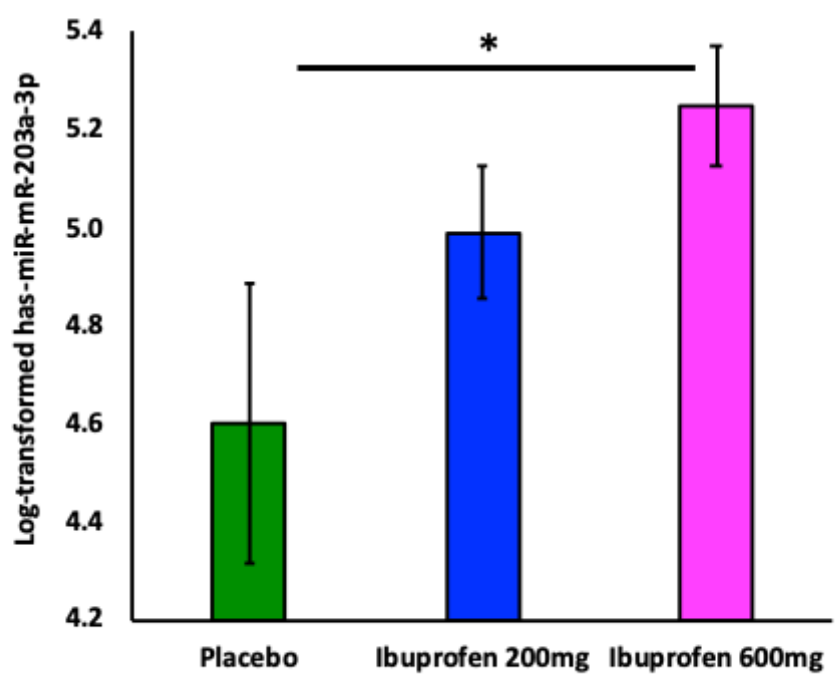

\section{Figure 5}

Ibuprofen-mediated effect on NEE MiRs ( $n=20)$. Log-transformed data shows ibuprofen-mediated (200 and $600 \mathrm{mg}$ ) effects on neuronally-enriched exosomal miRNA expression for A. miR-27b-3p; B. miR-320b; C. miR-23b-3p. Only $600 \mathrm{mg}$ ibuprofen differed for D. miR-203a-3p. * denotes significant differences between groups using paired t-test. 

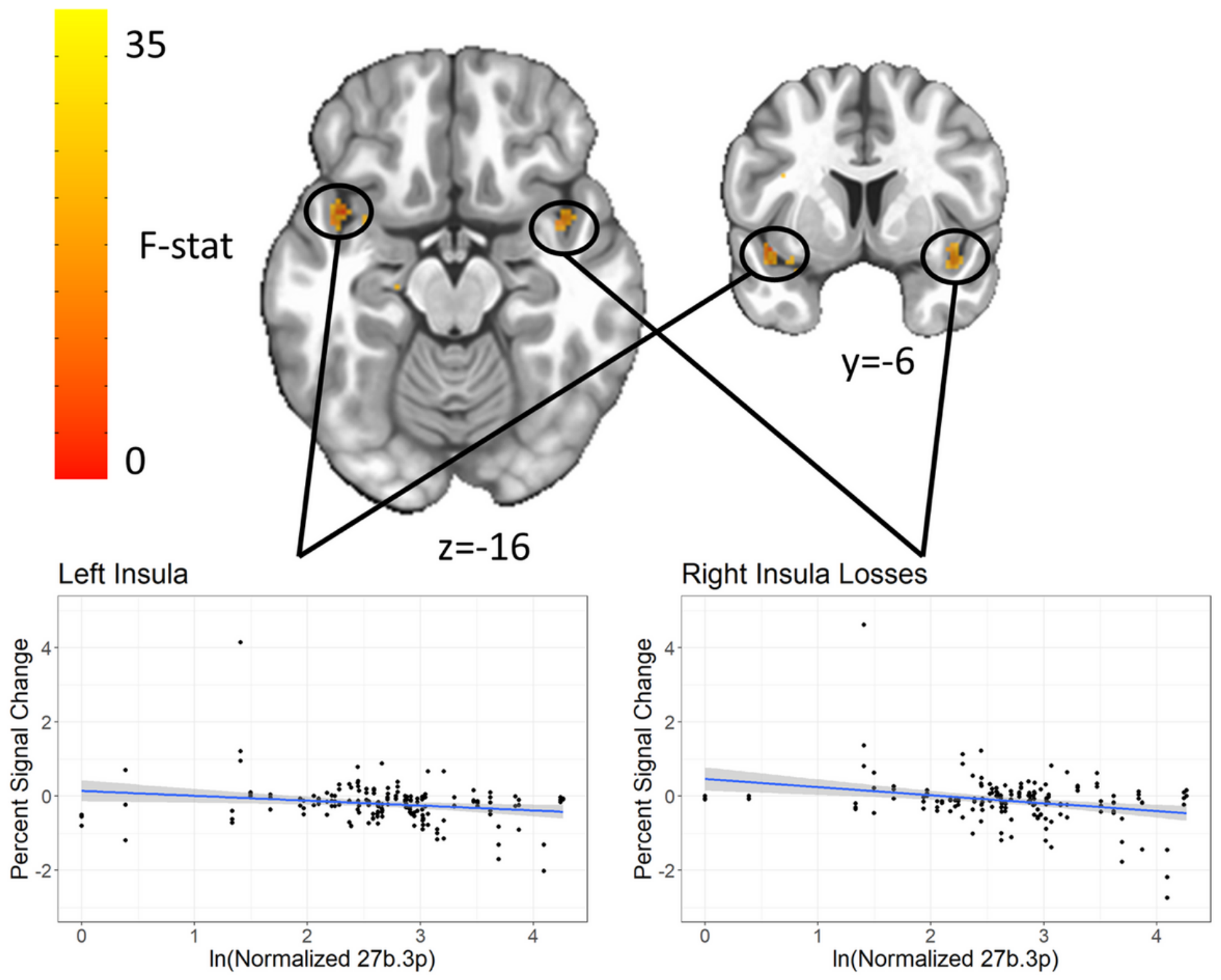

Figure 6

MiR-27b-3p Main Effect in Bilateral Insula for Loss Anticipation. Both the left and right ventral anterior insula show a main effect of levels of miR $27 \mathrm{~b}-3 \mathrm{p}$ in response to losses, where increased miR was related to decreased activation. Clusters were extracted with a voxelwise $p<0.001$ and clusterwise $a<0.01$. 

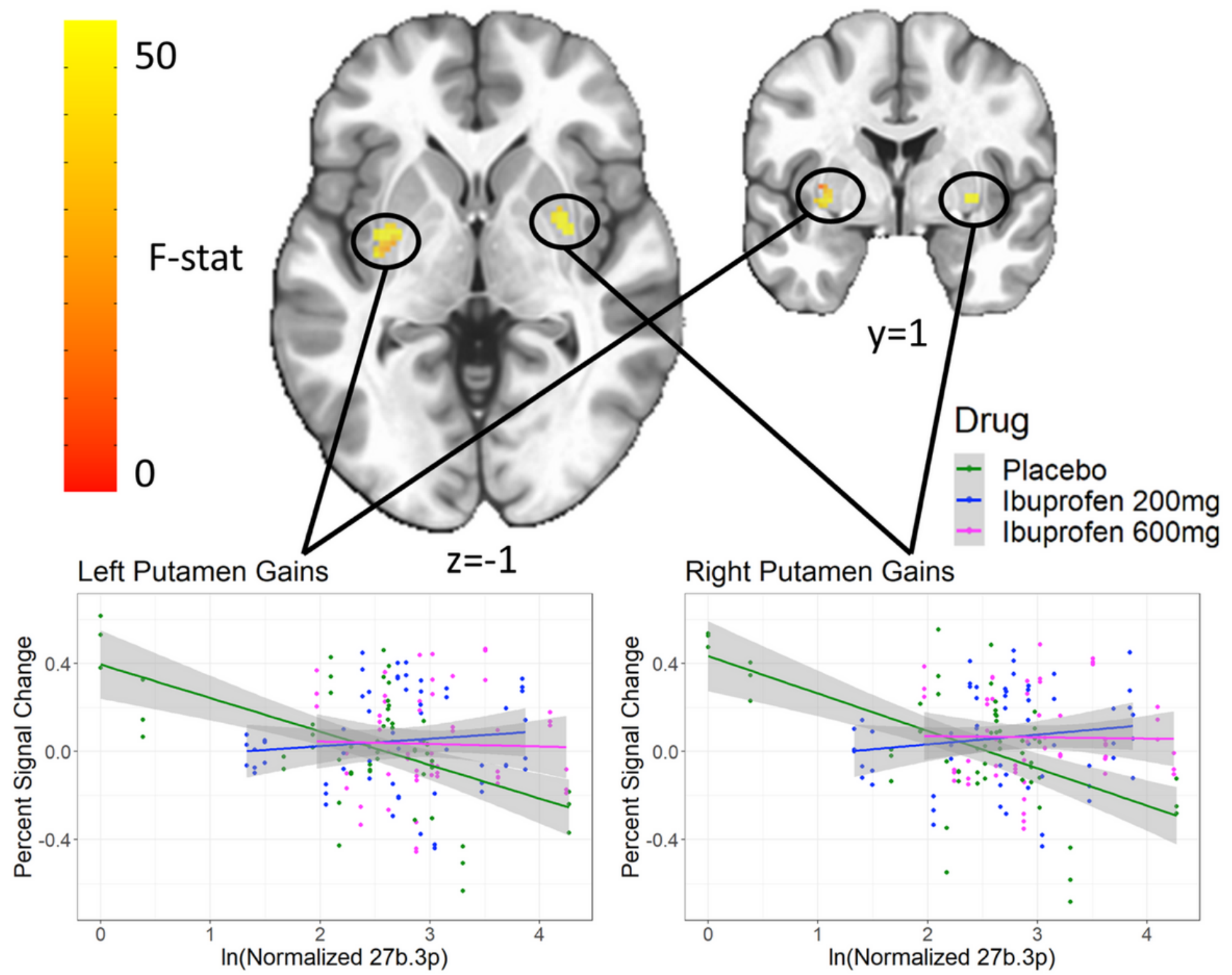

Figure 7

miR-27b-3p by Drug Interaction in Bilateral Putamen for Gain Anticipation. Both the left and right putamen show an interaction between levels of miR $27 \mathrm{~b}-3 p$ and drug in response to gains. Clusters were extracted with a voxelwise $p<0.001$ and clusterwise $a<0.01$.

\section{Supplementary Files}

This is a list of supplementary files associated with this preprint. Click to download.

- IBUNDEPaperSupplementalMaterials20210812.pdf 